\title{
Using machine learning to explore core risk factors associated with the risk of eating disorders among non-clinical young women in China: A decision-tree classification analysis
}

\author{
Yaoxiang Ren ${ }^{1}$, Chaoyi Lu ${ }^{2,3}$, Han Yang ${ }^{2,3}$, Qianyue Ma ${ }^{3}$, Wesley R. Barnhart ${ }^{4}$, Jianjun Zhou ${ }^{3}$ and Jinbo He ${ }^{1^{*}}$ (D)
}

\begin{abstract}
Background: Many previous studies have investigated the risk factors associated with eating disorders (EDs) from the perspective of emotion regulation (ER). However, limited research has investigated interactions between co-existing risk factors for EDs, especially in China where research in EDs is underrepresented.

Methods: This study examined core risk factors related to maladaptive eating behaviors and ER, and how their interactions affect the detection of EDs. Using machine learning, a decision tree model was constructed on a data set of 830 non-clinical Chinese young women with an average age of 18.91 years $(S D=0.95)$. The total data set was split into training and testing data sets with a ratio of 70 to $30 \%$.

Results: Body image inflexibility was identified as the major classifier for women at high risk of EDs. Furthermore, interactions between body image inflexibility, psychological distress, and body dissatisfaction were important in detecting women at high risk of EDs. Overall, the model classifying women at high-risk for EDs had a sensitivity of 0.88 and a specificity of 0.85 when applied to the testing data set.

Conclusions: Body image inflexibility, psychological distress, and body dissatisfaction were identified as the major classifiers for young women in China at high risk of EDs. Researchers and practitioners may consider these findings in the screening, prevention, and treatment of EDs among young women in China.
\end{abstract}

Keywords: Eating disorders, Machine learning, Decision tree, Risk factors, Chinese women

\section{Plain English summary}

Previous studies have identified multiple risk factors of eating disorders that are related to emotion regulation and coping strategies in the Western context. However, most of these studies failed to describe any kind of hierarchy or interaction between risk factors that co-occur. To address this knowledge gap, the present study investigated a broad range of risk factors from the perspective of emotion regulation and then used a decision tree classification method to screen for EDs among young women in China. Results showed that body image inflexibility, psychological distress, and body dissatisfaction were the primary classifiers for Chinese women at high risk of EDs.

*Correspondence: anlfhe@gmail.com; hejinbo@cuhk.edu.cn

${ }^{1}$ School of Humanities and Social Science, The Chinese University of Hong Kong, Shenzhen 518172, Guangdong, China

Full list of author information is available at the end of the article

\section{Background}

Eating disorders (EDs), mental illnesses that involve disturbances in body image and eating behaviors, are prevalent globally and represent a threat to public health $[1,2]$. original author(s) and the source, provide a link to the Creative Commons licence, and indicate if changes were made. The images or other third party material in this article are included in the article's Creative Commons licence, unless indicated otherwise in a credit line to the material. If material is not included in the article's Creative Commons licence and your intended use is not permitted by statutory regulation or exceeds the permitted use, you will need to obtain permission directly from the copyright holder. To view a copy of this licence, visit http://creativecommons.org/licenses/by/4.0/. The Creative Commons Public Domain Dedication waiver (http://creativeco mmons.org/publicdomain/zero/1.0/) applies to the data made available in this article, unless otherwise stated in a credit line to the data. 
Disordered eating behaviors, ranging from severe food restriction (as evidenced in anorexia nervosa) to binge eating (as evidenced in bulimia nervosa and binge eating disorders), can result in serious health consequences, disability, and even death [3]. The prevalence of EDs more than doubled between 2005 and 2018, from 3.5 to $7.8 \%$ $[4,5]$. Though research on EDs in Asian countries did not begin until the 1990s, it has since become a recognized form of psychopathology among young Asian females [6] with prevalence rising over time, especially in China [7, 8]. A recent national study showed that the prevalence of EDs is $7.04 \%$ in females aged $12-50$ years in China, which is comparable to other global regions [9]. Given China's large population base, more attention should be paid to its rate of EDs, especially in those individuals who are known to be at high risk.

Adolescent girls and young women have the highest risk of developing EDs $[10,11]$. For example, Yao and colleagues [9] found that those aged 21-25 had the highest prevalence of EDs in China. Consistent with this, Stice and colleagues [11] claimed that the peak age of onset of EDs in women is 16-20 years. Ample evidence has shown that females in this age group experience more vulnerabilities [12] associated with EDs, such as bodyrelated social comparison [13], body dissatisfaction [14], emotional difficulties [15], and psychological distress [5].

A growing body of research has focused on emotion regulation (ER) theory [16-18] and the effects of maladaptive coping strategies on eating behaviors $[19,20]$. For example, loss of control over eating, a core symptom of bulimia nervosa and binge-eating disorder, is considered a strategy for coping with negative emotions [21]. More specifically, people who lose control over eating tend to think that eating can distract them from or comfort them in response to negative emotions [22]. Emotional overeating, defined as overeating in response to negative emotions, has been found to be a strong predictor of overeating-related symptoms (e.g., binge eating) in EDs [23]. Taken together, this evidence suggests that individuals who lack ER skills may develop maladaptive eating behaviors in response to negative emotions, thus contributing to the development and maintenance of EDs [24]. From this perspective, well-recognized risk factors for EDs such as body dissatisfaction and psychological distress $[25,26]$ may further contribute to negative emotions and trigger disordered eating behaviors through maladaptive strategies such as emotional overeating and loss of control over eating [27].

Symptoms of anorexia nervosa involve difficulty in dealing with emotional responses (e.g., fear) to weight gain, and preoccupation with food restriction and weight control [28]. These symptoms are closely related to the concept of psychological inflexibility (i.e., the inability to behave flexibly [29]), and some research has characterized anorexia nervosa as a disorder of psychological inflexibility [30]. Two specific types of psychological inflexibility have been proposed: body image inflexibility [31] and eating inflexibility [32]. Body image inflexibility refers to the unwillingness to experience negative thoughts and emotions about one's appearance [33]. In a recent longitudinal study, body image flexibility emerged as the strongest predictor of ED psychopathology [34]. Other research has found that the link between disordered eating cognitions (e.g., fear of weight gain) and ED symptoms is established, in part, through body image inflexibility [35]. Eating inflexibility refers to an eating pattern that rigidly adheres to eating-related rules without respecting internal needs [36]. It has been shown to be significantly and positively associated with EDs, dietary restraint, picky eating, body image inflexibility, and overall psychological distress [32, 37].

Given the cross-cultural vulnerability of young females to develop EDs, research exploring new methodologies is needed to explain and stratify the various levels of risk among this group. The existing literature mainly uses regression-based methodologies, which involve rigid assumptions (e.g., linearity, homoscedasticity, independence, and normality) $[38,39]$ that are usually not met in studies of eating psychopathology (e.g., normality [4042]). In other words, regression-based methodologies may be inappropriate when there are non-linear relationships or a large number of potential interactions among risk factors, making the predictive power of each factor on ED risk unclear. It is therefore recommended that researchers pay attention to the concurrency and interactions among risk factors [43] as a way to try to understand psychopathology as an effect of all factors working together [44].

To address the concurrency and interactions among variables, machine learning has been used to explore the risk factors for several mental disorders [45-47]. In a 2-year longitudinal study, Haynos and colleagues [47] demonstrated the usefulness of machine learning in predicting ED symptoms. Specifically, decision tree-based models can provide a sequential and hierarchical combination of variables with which to optimize a screen, thereby avoiding the introduction of researcher bias [47, 48].

The existing literature contains few studies of disordered eating that use machine learning methods, and those that do exist were conducted in Western societies. For example, Linardon and colleagues [48] explored the roles of three different eating patterns (e.g., rigid, flexible, and intuitive) in predicting recurrent binge eating and found intuitive eating to be the most important factor. Another study used a decision tree method to predict 
the onset of EDs and found that BMI, overeating, and body dissatisfaction were the three strongest predictors of EDs [43]. However, to the best of our knowledge there has been no machine learning-based research that included eating or body image inflexibility as independent variables or considered the relationship between ER and maladaptive eating behaviors. Furthermore, there is no research to date using machine learning to explore ED risk factors among Chinese women. Considering that culture is significant to the cause and expression of EDs [49], more research should be conducted in China. Thus, by using a machine learning approach, the present study aimed to fill this gap in the literature by including a range of well-established ED risk factors from the ER perspective to identify young women in China who might be at high risk of EDs.

\section{Methods}

\section{Participants and procedure}

The data used in the current study were from a project approved by the Institutional Review Board of the Chinese University of Hong Kong, Shenzhen, involving participants recruited from Hunan Agriculture University. The survey was conducted using paper and pencil. Psychology teachers at the university introduced the study to their 1412 first- and second-year undergraduate students during class time and invited them to participate. During data collection, all participants provided informed consent and were reminded of survey independence and authenticity. In addition, two attention check questions were used to ensure the quality of the responses. After removing those who did not provide informed consent or failed the two attention check questions, a total of 1,065 undergraduate students were eligible. Of these, our study focused on the women ( $n=830,77.9 \%)$, the ages of whom ranged from $18-23$ years $($ mean $=18.91, S D=0.95)$.

\section{Measures}

\section{Independent variables}

The present study aimed to include a range of ER-related risk factors to identify young women in China at high risk of EDs.

Body Mass Index (BMI) BMI was derived from selfreported height and weight and ranged from 15.22 to $31.22 \mathrm{~kg} / \mathrm{m}^{2}$ with a mean of $20.16 \mathrm{~kg} / \mathrm{m}^{2}(S D=2.39)$.

Psychological distress The Chinese version of the 6-item Kessler Scale (K6) was used to assess psychological distress. The scale consists of 6 items on a 5 -point Likert scale from 0 (all of the time) to 4 (none of the time) in regards to psychological distress [50]. Sample items include "During the past 4 weeks, how much of the time did you feel hope- less?" The Cronbach's $\alpha$ coefficient (i.e., internal consistency reliability; 0.84 ) and the test-retest Spearman correlation coefficient (0.79) have been shown to be acceptable in Chinese undergraduate students [51]. The Cronbach's $\alpha$ of the $\mathrm{K} 6$ in the current study was 0.90 .

Eating inflexibility To assess eating inflexibility, the Chinese version of the Inflexible Eating Questionnaire (C-IEQ) was used. The IEQ consists of 11 items on a 5 -point Likert scale from 1 (fully disagree) to 5 (fully agree). Sample items include "When I cannot follow my eating plan, I feel very anxious (or nervous)." [32]. The Chinese version of the IEQ [52] demonstrated acceptable internal consistency reliability with a Cronbach's $\alpha$ of 0.87 in undergraduate students [53]. Furthermore, significant correlations between the C-IEQ and other related constructs (e.g., ED symptomatology, body image inflexibility) indicated convergent validity of this measure [52]. The Cronbach's $\alpha$ of the C-IEQ in the current study was 0.90 .

Body image inflexibility Body image inflexibility was assessed by the Chinese version of the Body Image Acceptance and Action Questionnaire short form (C-BIAAQ-5). The BI-AAQ-5 is a 5-item abbreviated scale of the 12-item BI-AAQ [54]. Statements such as "Worrying about my weight makes it difficult for me to live a life that I value" were evaluated with a 7-point Likert scale from 1 (never true) to 7 (always true) [54]. Similar to previous studies of body image inflexibility $[55,56]$, to calculate the total score we averaged the item responses rather than reverse scoring all items and then averaging the responses. In previous research, internal consistency reliability was acceptable (Cronbach's $\alpha=0.89$ ), and strong correlations with related constructs (e.g., psychological inflexibility and body dissatisfaction) showed convergent validity in undergraduate students [57]. The Cronbach's $\alpha$ of the C-BI-AAQ-5 in the current study was 0.92 .

Body dissatisfaction The body dissatisfaction subscale of the Eating Disorder Inventory (EDI-BD) was used to assess body dissatisfaction. The EDI-BD contains 9 items such as "I think that my thighs are too large." Items are rated on a 6-point scale from 1 (never) to 6 (always) [58]. The Chinese Version of EDI demonstrated acceptable reliability and validity in undergraduate students [59]. In the current study, Cronbach's $\alpha$ for the EDI-BD subscale was 0.89 .

Emotional overeating The Emotional Overeating subscale of Chinese Adult Eating Behavior Questionnaire (C-AEBQ) was used to measure emotional overeating. It contains 5 items such as "I eat more when I am upset" which were evaluated on a 5 -point Likert scale from 1 
(fully disagree) to 5 (agree). Previous studies showed the C-AEBQ had acceptable internal consistency reliability and convergent and divergent validity in Chinese undergraduate students [60]. Cronbach's $\alpha$ of the Emotional Overeating subscale in the current study was 0.94 .

Loss of control over eating The Chinese version of Loss of Control Over Eating Scale-Brief (C-LOCES-B) was used to assess loss of control over eating. The LOCES-B contains 7 items from the 24-item LOCES, both of which have been validated with Cronbach's $\alpha$ of 0.96 and 0.93 , respectively [21]. Statements such as "I felt helpless about controlling my eating" were scored on a 5-point Likert scale ranging from 1 (never) to 5 (always). The C-LOCES$B$ showed acceptable internal consistency reliability with a Cronbach's $\alpha$ of 0.92 in Chinese undergraduate students [61]. Cronbach's $\alpha$ of the C-LOCES-B in the current study was 0.92 .

\section{Dependent variable}

Risk of eating disorders The Chinese version of Short Form of the Eating Disorder Examination Questionnaire (EDE-QS) [62] was used to measure the risk of EDs. The EDE-QS consists of 12 items with response options ranging from 0 to 3 days within the past 7 days [63]. The Chinese version of EDE-QS showed good psychometric properties in Chinese university students [62]. In the current study, Cronbach's $\alpha$ of the EDE-QS was 0.90. A total score of 15 or above on the EDE-QS showed good sensitivity and specificity to screen individuals with EDs [64]. In the current sample, 133 females (16\%) had EDE-QS scores of 15 or above, and thus were considered at high risk of developing EDs.

\section{Data analytic strategy Dealing with missing values}

In these data, the percentage of missing values across study variables ranged from $0.12 \%$ (loss of control over eating) to $2.29 \%$ (eating inflexibility). To make full use of the dataset, these missing values were handled by imputation rather than abandonment. Imputation of missing values was performed by IterativeImputer [65] with the fancyimpute package [48] of Python. The missing values were replaced with predictions from regression, and the imputation process was performed for each feature in an iterative fashion for a given number of rounds. The results of the final imputation round are returned.

\section{Balancing data}

Our data were imbalanced with a smaller proportion of positive cases $(16.02 \%, 133$ out of 830 samples) than negative ones (83.98\%, 697 out of 830 samples). Directly training a model with imbalanced data can result in good performance for the majority class but poor performance for the minority class. For the present dataset, it would result in a model that underperforms when applied to groups at high risk of EDs $[66,67]$. To handle imbalanced data in our classifications, we adopted data-level approaches, such as over- and under-sampling methods, which preprocess the imbalanced data before model training begins. The synthetic minority oversampling technique (SMOTE) is one such approach that has been widely used to enhance model performance in classification tasks with imbalanced data $[68,69]$. SMOTE, as proposed by Chawla and colleagues [70], creates synthetic data for the minority class. It works by finding k-nearest neighbors from random data in the minority class and then synthesizes new data between the randomly picked data and its k-nearest neighbors. It has also proven efficient at improving model performance in decision tree models $[71,72]$. Therefore, after splitting the training and testing data, the training set was balanced with SMOTE prior to model training.

In our task, $70 \%$ of the data $(n=581$ of 830$)$ was used for training and $30 \%(n=249$ of 830$)$ was used for testing. The original training dataset had 93 (16.01\%) positive samples and 488 (83.99\%) negative samples. After SMOTE, 395 synthetic positive samples were added to the training set to yield a final $n$ equal to that of the negative samples.

\section{Decision tree construction}

We performed decision tree classification using the sklearn package of Python [73]. The decision tree algorithm operates as follows. First, all samples are contained in a root node, which is then partitioned according to the best attribute (selected using Gini or Entropy). After selecting the best attribute, the algorithm then selects a split point that will maximize the purity of the resulting groups, and the dataset is partitioned into two new nodes. For each new node, the procedure is repeated recursively until no further splitting can improve the model's predictive accuracy [74]. Some branches in the tree may even be pruned (removed) to improve predictive accuracy.

The development of a decision tree model with preprocessed data can be divided into two parts: model training and model testing. Before training, all the samples are first randomly split into two subsets, training and testing, with a defined size ratio. In the present study the size ratio was set at 7:3 (training to testing). The decision tree classification algorithm was applied to the training set to build the model. The tree was then pruned to avoid overfitting these data, and the maximum number of leaf nodes allowed in the tree was restricted. By 
limiting the number of leaf nodes, the number of attributes selected in the tree and the depth of the tree can be well controlled.

Model testing is the evaluation of the model's performance when applied to the testing dataset. Each sample in the testing set was tested against the decision tree, tracing a path from the root to a specific leaf node which returned the predicted class for the sample [75]. If the predicted class for a sample was the same as the actual class, then the prediction was scored as correct for that sample. Sensitivity of the model was determined as the proportion of positive tuples (samples classified at high risk of EDs) that were correctly identified, and specificity was the proportion of negative tuples (samples classified at low risk of EDs) that were correctly identified.

\section{Results}

\section{Preliminary analyses}

Descriptive statistics for the entire dataset and ED risk classifications are presented in Table 1. Pearson correlations among the independent variables are presented in Additional file 1: Table S1. The following features were found at higher rates among samples classified as highrisk than in those classified as low-risk: higher BMI, eating inflexibility, psychological distress, loss of control over eating, body image inflexibility, body dissatisfaction, and emotional overeating.

\section{Decision tree classification model construction}

Body image inflexibility, psychological distress, and body dissatisfaction were identified as important classifiers for identifying individuals at high risk of EDs (i.e., having EDE-QS scores equal to or greater than 15). As an indicator of relative importance between classifiers, feature importance was then calculated for each of the three major classifiers. Feature importance scores were 0.81, 0.15 , and 0.04 , for body image inflexibility, psychological distress, and body dissatisfaction, respectively.

As shown in Fig. 1, participants were first partitioned by body image inflexibility scores as measured by the BIAAQ-5. Participants with higher body image inflexibility scores $(>15.01)$ constituted $47.4 \%$ of the overall training samples, and $84 \%$ of these participants were classified at high risk of EDs. These participants were further partitioned by psychological distress scores as measured by the K6. Those with higher psychological distress scores (>5.06) were further classified at high risk of EDs. Those with lower psychological distress scores $(\leq 5.06)$ were further partitioned by body dissatisfaction scores as measured by the EDI-BD. Among these participants, those with higher body dissatisfaction scores ( $>45.26)$ tended to be at high risk of EDs, while the others were not.

Participants with lower body image inflexibility scores $(\leq 15.01)$ were partitioned and classified in a different way. Those with lower psychological distress scores ( $\leq$ 7.02) were at low risk of EDs. Individuals with higher scores $(>7.02)$ were further partitioned by body image inflexibility. Those with lower body image inflexibility scores $(<11.03)$ were at low risk of EDs, while those with relatively higher body image inflexibility scores $(>11.03$ and $\leq 15.01$ ) were at high risk.

Overall, participants with higher body image inflexibility scores, higher psychological distress scores, and/or higher body dissatisfaction scores were more likely to be categorized at high risk of EDs.

\section{Decision tree classification model evaluation}

Similar to a previous study [48], the present model was evaluated according to its sensitivity and specificity. Sensitivity reflects the probability that the model will

Table 1 Comparison of groups on study variables

\begin{tabular}{|c|c|c|c|c|c|c|c|}
\hline \multirow[b]{3}{*}{ Variable } & \multicolumn{7}{|c|}{ Eating disorder risk classification } \\
\hline & \multicolumn{2}{|c|}{ Total sample $(n=830)$} & \multicolumn{2}{|c|}{ No $(n=697)$} & \multicolumn{2}{|c|}{ Yes $(n=133)$} & \multirow[b]{2}{*}{$d[95 \% \mathrm{Cl}]$} \\
\hline & $M$ & $S D$ & $M$ & $S D$ & $M$ & $S D$ & \\
\hline BMI & 20.16 & 2.40 & 20.04 & 2.41 & 20.82 & 2.20 & $0.33[0.14,0.51]$ \\
\hline Eating inflexibility & 30.70 & 7.11 & 29.91 & 6.87 & 34.88 & 6.91 & $0.72[0.53,0.91]$ \\
\hline Psychological distress & 11.71 & 4.32 & 11.00 & 3.87 & 15.45 & 4.64 & $1.11[0.92,1.31]$ \\
\hline Loss of control over eating & 13.34 & 5.51 & 12.34 & 4.88 & 18.59 & 5.68 & $1.25[1.05,1.45]$ \\
\hline Body image inflexibility & 11.59 & 6.30 & 10.11 & 5.10 & 19.35 & 6.29 & $1.74[1.53,1.95]$ \\
\hline Body dissatisfaction & 34.60 & 9.13 & 33.61 & 8.79 & 39.79 & 9.16 & $0.70[0.51,0.89]$ \\
\hline Emotional over eating & 2.18 & 0.92 & 2.06 & 0.88 & 2.83 & 0.87 & $0.88[0.69,1.07]$ \\
\hline
\end{tabular}

$M=$ mean, $S D=$ standard deviation, $d=$ Cohen's $d, C l=$ confidence interval. The classification of "Yes" stands for groups classified with high risk of EDs, and "No" stands for groups classified with low risk of EDs 


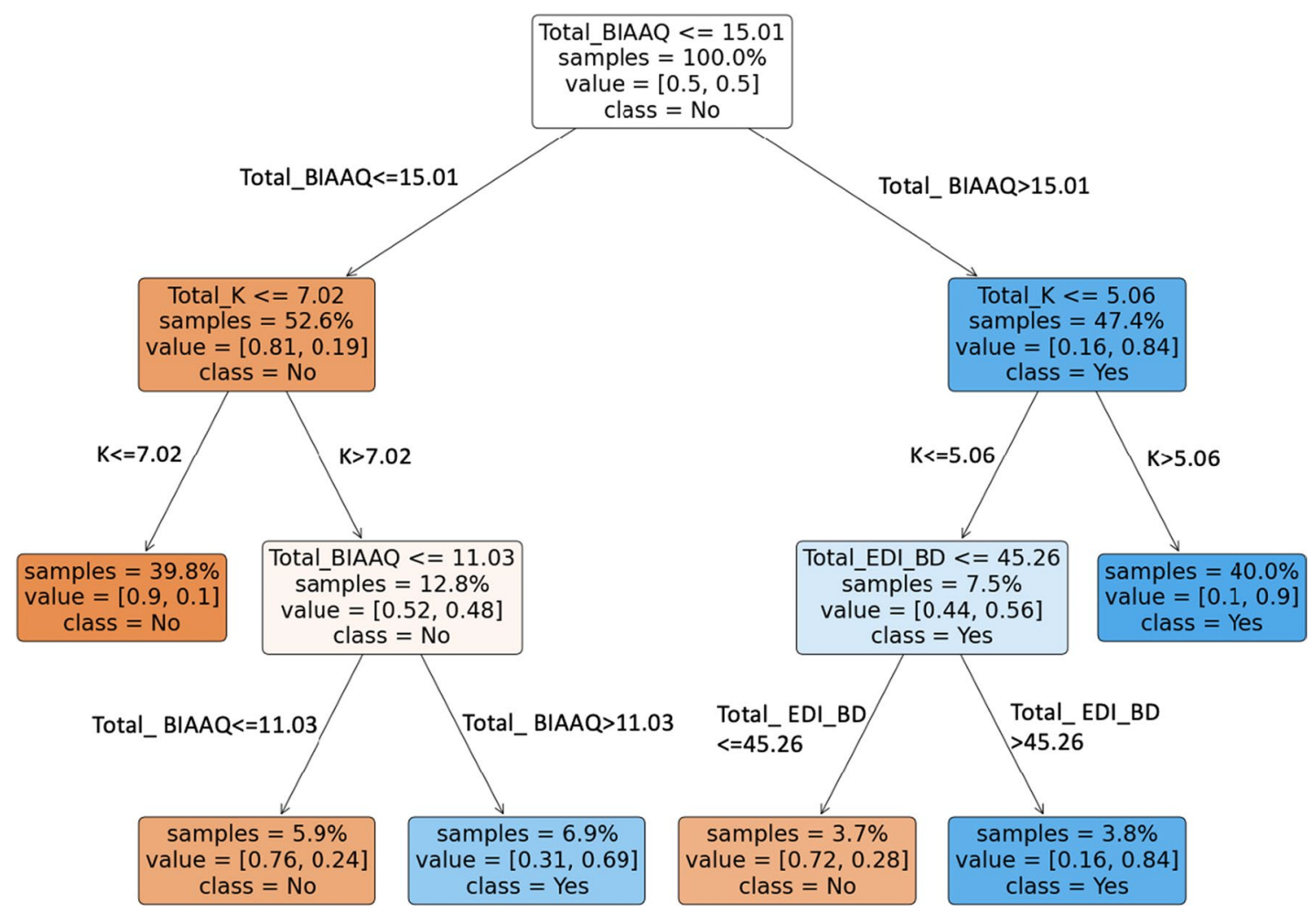

Fig. 1 Decision tree for classifying at-risk of EDs. Note: Figure shows the classification tree for at-risk of EDs based on the training subsample of the overall dataset $(n=581$ of 830). Total_BIAAQ = total score of Body Image Acceptance and Action Questionnaire. Total_K=total score of Kessler Scale to assess psychological distress. Total_EDI_BD = Body Dissatisfaction subscale of the Eating Disorder Inventory. For each internal node, the first line refers to a decision rule with a selected attribute. For example, the root node indicates a decision rule that the attribute body image inflexibility is smaller than or equal to 15.01. For a node with branches, its left child node follows the decision rule in the parent node, whereas its right child follows the complement of the decision rule. The second line of each internal node indicates the percentage of samples involved in this node. The third line refers to the percentages of positive samples (i.e., samples at-risk of EDs) and that of negative samples (i.e., samples without at-risk of EDs) within each node. The shade of color refers to the purity of each node, implying the extent of a mixture of groups for a subset of samples. The dark color means most samples belong to one group. Lastly, class in each box indicates whether high risk of EDs is more prevalent in a node. Blue boxes with class = yes indicate at-risk of EDs is more prevalent, whereas orange boxes with class = no indicate the subgroups contain more people with low risk of EDs, based on EDE-QS scores

correctly include individuals at high risk of EDs, and specificity reflects the probability that it will correctly exclude those that are not at high risk of EDs. When classifying individuals from the test subset of data $(n=249$ of 830) the model had a sensitivity of 0.88 and a specificity of 0.85 . That is, the model performs slightly better in correctly identifying people at high risk of EDs $(88 \%, n=40)$ than for classifying those not at high risk $(85 \%, n=209)$. To further validate the evaluation results across the entire dataset, a random sampling method was adopted. By randomly selecting $30 \%$ of the overall dataset, five different times, the average sensitivity was 0.85 and the average specificity was 0.86 , which aligns with the evaluation of the test subsample.

Regarding model complexity, seven attributes were initially taken into consideration and the model ultimately selected three of them. The minimum number of samples contained in a single node was 36 (3.7\% of 976 training samples, including 395 synthetic training samples and 581 original training samples). Moreover, we generated 50 different test sets by changing the random seed used to split the training and test sets and used these to further evaluate the proposed model. The mean sensitivity across the 50 test sets was $0.84(S D=0.05)$ and mean specificity was $0.86(S D=0.02)$. As shown in Fig. 2, these results demonstrate the sufficiency of our samples for yielding a stable model.

\section{Discussion}

With machine learning, the current study used a decision tree to identify young women in China who may be at high risk of developing an ED. The strengths of this study include its ER framework to examine several common risk factors for EDs in a non-clinical sample of 


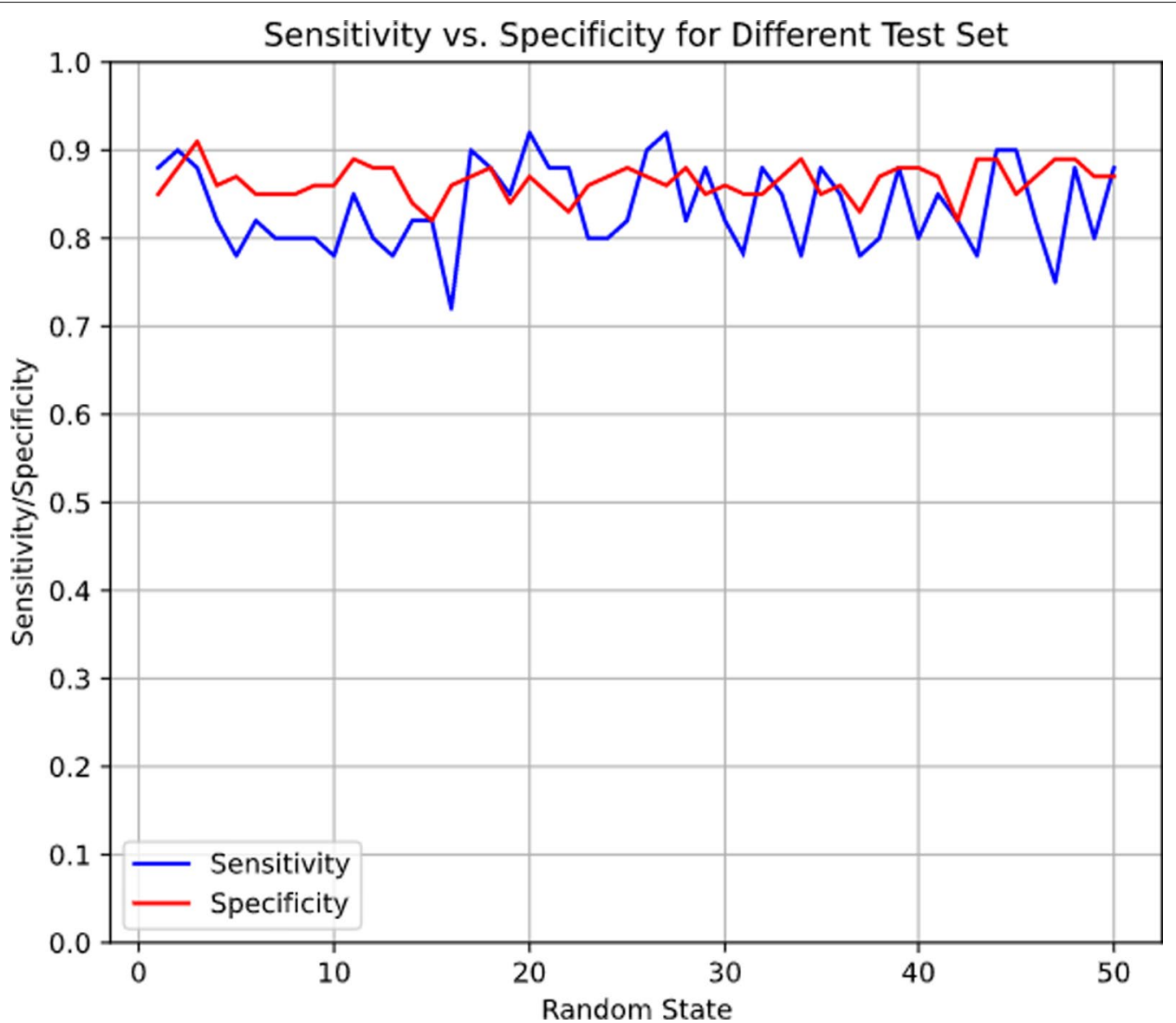

Fig. 2 Sensitivity and specificity for different test set. Note: The random state determines the randomness in the split of training set and test set. Therefore, different random states correspond to different test sets. Our proposed model was evaluated by each test set and the test sensitivity and specificity were recorded for each test set. In the figure above, the blue curve represents the change of test sensitivity with different test sets and the red curve represents the change of test specificity with different test sets

young women in China. Furthermore, the decision tree method allows for deeper exploration of these variables concurrently and how they may interact in relation to ED risk among young women in China. The findings add to our understanding of the role of body image inflexibility in detecting EDs in the Chinese context, which is critical because most existing research has been conducted in Western cultures. Furthermore, distinctive interactions between the major classifiers show that in young women with a high level of body image inflexibility, elevated psychological distress could amplify the risk of EDs. These findings further suggest that increasing body image flexibility may improve tolerance to psychological distress and thus lower the risk of EDs among young Chinese women.

The present findings have several implications for the intervention and prevention of EDs in non-clinical young women in China. First, body image inflexibility was the most significant factor for detecting EDs in the current sample. This is consistent with prior studies in the United States that demonstrated a key role of body image flexibility and body image inflexibility in the development of EDs [35, 76]. For example, intervention studies indicated that body image flexibility was the strongest predictor of ED symptomatology $[34,76]$. In addition, as body image flexibility and body image inflexibility have been proposed as variables linking negative emotion [77], negative body image [78], and negative cognitions [35] with EDs, it seems necessary to incorporate body image inflexibility with theoretical models under emotion and cognition frameworks to better understand the psychopathology of EDs. Body dissatisfaction was rated less important than body image inflexibility or psychological distress by the present model; thus, future research into ED prevention and intervention should prioritize body image flexibility/inflexibility and psychological distress. This is in line with the common practice of treating EDs with Acceptance and Commitment Therapy, in which body image 
flexibility is promoted as a major adaptive emotional mechanism to reduce ED psychopathology [79].

When introducing psychological distress into the decision tree model, the effects and interactions between psychological distress and body image inflexibility were found to be distinctive. Of the group with high body image inflexibility and high psychological distress, 90\% were found to be at high risk of EDs, indicating that elevated psychological distress may amplify the risk of EDs in individuals who have a high level of body image inflexibility. On the other hand, participants with low body image inflexibility and psychological distress were classified as having low risk of EDs. Comparing the two thresholds of psychological distress under different levels of body image inflexibility, the present findings suggest that a lower level of body image inflexibility may reduce the impact of psychological distress on ED risk: those with low body image inflexibility were generally at lower risk of EDs regardless of the level of psychological distress. This makes sense given that greater body image flexibility can help people accept negative emotions and thoughts about their bodies, which may in turn promote more adaptive behaviors when dealing with such emotions [80]. Previous research has shown a negative correlation between psychological flexibility and disordered eating [81, 82], and a protective effect of psychological flexibility in relation to stress and mental health [83]. Still, more research is needed to probe the interaction between these factors and their role in EDs.

It is also worth noting that the group with high levels of psychological distress was further partitioned by body image inflexibility into two groups (with either high or low risk of EDs), indicating that mid-level body image inflexibility concurrent with high-level psychological distress may also be associated with high risk of EDs. Nuanced interactions such as this could provide a framework for clinical practice involving a variety of mental health conditions. For example, practitioners may focus on reducing both body image inflexibility and psychological distress for individuals with high body image inflexibility, whereas they may focus more on targeting psychological distress for individuals with low body image inflexibility. More research is needed to investigate the interactions and thresholds of these two factors in other datasets and using different intervention outcomes.

As a third-level classifier, body dissatisfaction separates those with high body image inflexibility and low psychological distress into two further nodes. In the high node (body dissatisfaction $>45.26$, as compared to the sample mean of 34.60 ), $84 \%$ of the group were classified as highrisk. This is consistent with numerous studies showing positive correlations between body dissatisfaction and ED psychopathology $[14,84]$, although the sample size identified by this node was relatively small (only $3.8 \%$ of the total sample). Even though it is a well-acknowledged risk factor for EDs, body dissatisfaction contributed less to the current decision tree model than expected; its feature importance was only $4 \%$. This differed from the results of another machine learning-based study showing that body dissatisfaction was the most important classifier of EDs among variables including, but not limited to, depressive symptoms, negative affectivity, and dietary restraint [11]. A reason why body dissatisfaction was less important in the current model could be explained by interaction effects between body dissatisfaction and body image inflexibility. Body image inflexibility has been identified as a mediator between body dissatisfaction and eating disorder symptoms in several studies [78, 85]. In mediation analysis, the direct effects of body dissatisfaction on disordered eating dropped significantly after adding body image inflexibility as a mediator [85]. Thus, analogous to the effects in the mediation model, it could be that the inclusion of body image inflexibility weakens the predictive power of body dissatisfaction in the current decision tree model. However, this hypothesis should be tested in samples from other cultures or populations.

The present findings may be beneficial for practitioners to understand and intervene in ED symptomatology in non-clinical young women in China. For instance, when designing large-scale and cost-effective prevention/intervention programs to reduce ED symptomatology among young women in China, targeting factors may be prioritized to include the three risk factors highlighted in the current study. However, caution should be taken when interpreting the present findings. First, to reduce model complexity, some of our initial variables were not used in the final decision tree (BMI, emotional overeating, loss of control over eating, and eating inflexibility). This does not necessarily mean that these variables are not related to ED risk, but rather that they convey less explanatory and interactive power than others in this particular set of variables and data. Moreover, the results, especially the thresholds of the classifiers, should be considered provisional until validated by future studies. Finally, based on the sensitivity analysis (see Additional file 1) with another sample of non-clinical young women in which SCOFF [86] was used as the screening tool, the results consistently showed that body image inflexibility was the most important risk factor, supporting the robustness of the current findings on body image inflexibility.

The current study is not free from limitations. First, the sample only consisted of young women from a university population. Further exploration of larger and more diverse samples is needed to be able to generalize to different populations (e.g., adolescent girls and adult 
women). Second, the study's cross-sectional design limits our ability to draw conclusions about causal relationships between study variables. Well-designed prospective research is needed to confirm these exploratory findings. Thirdly, the EDE-QS can only screen participants at risk of EDs in general; in other words, it cannot distinguish specific EDs such as anorexia nervosa, bulimia nervosa, or binge-eating disorder. Thus, the current results cannot be generalized to patients with a specific ED diagnosis, and we expect studies involving patients with EDs (e.g., [87]) to further explore the risk factors of specific types of EDs in the Chinese context.

\section{Conclusions}

This is the first study to use a decision tree classification analysis in the Chinese context to explore interactions between ER-related factors and ED risk. Several conclusions were drawn from a decision tree built upon data with three major classifiers: body image inflexibility, psychological distress, and body dissatisfaction. Among them, body image inflexibility was most predictive of high ED risk, which is consistent with a number of other studies. Furthermore, novel interactions between body image inflexibility and psychological distress were found in relation to ED risk. These findings could be further extended to identify potentially high-risk groups in order to guide ED prevention measures among young Chinese women. Future studies are necessary to test the interactive hypotheses and improve prediction accuracy for the assessment, diagnosis, and intervention of EDs in the Chinese context.

\section{Abbreviations}

EDs: Eating disorders; ER: Emotion regulation; C-AEBQ: Chinese translation of the Adult Eating Behavior Questionnaire; IEQ: Inflexible Eating Questionnaire; EDI: Eating Disorder Inventory; BI-AAQ: Body Image Acceptance and Action Questionnaire; LOCES-B: Loss of Control Over Eating Scale-Brief.

\section{Supplementary Information}

The online version contains supplementary material available at https://doi. org/10.1186/s40337-022-00545-6.

Additional file 1. Pearson correlation analysis and sensitivity analysis.

\section{Acknowledgements}

The authors would like to thank all participants involved in this study.

\section{Authors' contributions}

YR drafted and revised the manuscript. CL and QM built the machine learning model, performed the statistical analysis, and helped draft and revise the manuscript. HY and JZ helped perform the statistical analysis and helped draft and revise the manuscript. WRB helped draft and revise the manuscript. JH led the study design and results interpretation and helped draft and revise the manuscript. All authors read and approved the final manuscript.
Funding

This research was supported by the Shenzhen Research Institute of Big Data.

\section{Availability of data and materials}

The datasets used and/or analyzed during the current study are available from the corresponding author on reasonable request.

\section{Declarations}

\section{Ethics approval and consent to participate}

This study received ethical approval from the institutional review board of the Chinese University of Hong Kong, Shenzhen, and informed consent was presented to all participants.

\section{Consent for publication}

Not applicable.

\section{Competing interests}

The authors declare that they have no competing interests.

\section{Author details}

'School of Humanities and Social Science, The Chinese University of Hong Kong, Shenzhen 518172, Guangdong, China. ${ }^{2}$ School of Data Science, The Chinese University of Hong Kong, Shenzhen, Guangdong, China. ${ }^{3}$ Present Address: Shenzhen Research Institute of Big Data, Shenzhen, Guangdong, China. ${ }^{4}$ Department of Psychology, Bowling Green State University, Bowling Green, Ohio, USA.

Received: 6 September 2021 Accepted: 27 January 2022

Published online: 10 February 2022

\section{References}

1. Hoek HW. Review of the worldwide epidemiology of eating disorders. Curr Opin Psychiatry. 2016;29(6):336-9. https://doi.org/10.1097/YCO. 0000000000000282.

2. Stice E, Becker CB, Yokum S. Eating disorder prevention: current evidencebase and future directions. Int J Eat Disord. 2013;46(5):478-85. https://doi. org/10.1002/eat.22105.

3. Erskine HE, Whiteford HA, Pike KM. The global burden of eating disorders. Curr Opin Psychiatry. 2016;29(6):346-53. https://doi.org/10.1097/YCO. 0000000000000276.

4. Galmiche M, Déchelotte P, Lambert G, Tavolacci MP. Prevalence of eating disorders over the 2000-2018 period: a systematic literature review. Am J Clin Nutr. 2019;109(5):1402-13. https://doi.org/10.1093/ajcn/nqy342.

5. Mitchell KS, Mazzeo SE. Binge eating and psychological distress in ethnically diverse undergraduate men and women. Eat Behav. 2004;5(2):15769. https://doi.org/10.1016/j.eatbeh.2003.07.004.

6. Smolak L, Levine MP. The Wiley handbook of eating disorders: Wiley Online Library. New York: Wiley; 2015. https://doi.org/10.1002/97811 18574089.

7. Pike KM, Dunne PE. The rise of eating disorders in Asia: a review. J Eat Disord. 2015;3(1):1-14. https://doi.org/10.1186/s40337-015-0070-2.

8. Wu J, Lin Z, Liu Z, He H, Bai L, Lyu J. Secular trends in the incidence of eating disorders in China from, 1990 to 2017: a joinpoint and age-periodcohort analysis. Psychol Med. 2020. https://doi.org/10.1017/S003329172 0002706.

9. Yao S, Zhang R, Thornton LM, Peat CM, Qi B, Du S, Wang H, Zhang B, Bulik CM. Screen-detected disordered eating and related traits in a large population sample of females in mainland China: China Health and Nutrition Survey. Int J Eat Disord. 2021;54(1):24-35. https://doi.org/10.1002/eat. 23409.

10. Nagl M, Jacobi C, Paul M, Beesdo-Baum K, Höfler M, Lieb R, Wittchen H-U. Prevalence, incidence, and natural course of anorexia and bulimia nervosa among adolescents and young adults. Eur Child Adolesc Psychiatry. 2016;25(8):903-18. https://doi.org/10.1007/s00787-015-0808-z.

11. Stice E, Marti CN, Durant S. Risk factors for onset of eating disorders: evidence of multiple risk pathways from an 8-year prospective study. Behav Res Ther. 2011;49(10):622-7. https://doi.org/10.1016/j.brat.2011.06.009. 
12. Duarte C, Ferreira C, Trindade IA, Pinto-Gouveia J. Normative body dissatisfaction and eating psychopathology in teenage girls: the impact of inflexible eating rules. Eat Weight Disord Stud Anorex Bulim Obes. 2016;21(1):41-8. https://doi.org/10.1007/s40519-015-0212-1.

13. Hamel AE, Zaitsoff SL, Taylor A, Menna R, Grange DL. Body-related social comparison and disordered eating among adolescent females with an eating disorder, depressive disorder, and healthy controls. Nutrients. 2012;4(9):1260-72. https://doi.org/10.3390/nu4091260.

14. Chen G, He J, Zhang B, Fan X. Revisiting the relationship between body dissatisfaction and eating disorder symptoms in Chinese adolescents: the mediating roles of regulatory emotional self-efficacy and depression symptoms. Eat Weight Disord Stud Anorex Bulim Obes. 2020;26:1-9. https://doi.org/10.1007/s40519-020-00848-0.

15. Shriver LH, Wollenberg G, Gates GE. Prevalence of disordered eating and its association with emotion regulation in female college athletes. Int J Sport Nutr Exerc Metab. 2016;26(3):240-8. https://doi.org/10.1123/ijsnem. 2015-0166.

16. Hill ML, Masuda A, Latzman RD. Body image flexibility as a protective factor against disordered eating behavior for women with lower body mass index. Eat Behav. 2013;14(3):336-41.

17. Brockmeyer T, Skunde M, Wu M, Bresslein E, Rudofsky G, Herzog W, Friederich HC. Difficulties in emotion regulation across the spectrum of eating disorders. Compr Psychiatry. 2014;55(3):565-71. https://doi.org/10.1016/j. comppsych.2013.12.001.

18. Harrison A, Sullivan S, Tchanturia K, Treasure J. Emotional functioning in eating disorders: attentional bias, emotion recognition and emotion regulation. Psychol Med. 2010;40(11):1887-97. https://doi.org/10.1017/ S0033291710000036.

19. Evers C, Marijn Stok F, de Ridder DT. Feeding your feelings: emotion regulation strategies and emotional eating. Pers Soc Psychol Bull. 2010:36(6):792-804. https://doi.org/10.1177/0146167210371383.

20. Goossens L, Braet C, Van Vlierberghe L, Mels S. Loss of control over eating in overweight youngsters: the role of anxiety, depression and emotional eating. Eur Eat Disord Rev. 2009;17(1):68-78. https://doi.org/10.1002/erv. 892.

21. Latner JD, Mond JM, Kelly MC, Haynes SN, Hay PJ. The loss of control over eating scale: development and psychometric evaluation. Int J Eat Disord. 2014;47(6):647-59. https://doi.org/10.1002/eat.22296.

22. Burton E, Stice E, Bearman SK, Rohde P. Experimental test of the affectregulation theory of bulimic symptoms and substance use: a randomized trial. Int J Eat Disord. 2007;40(1):27-36. https://doi.org/10.1002/eat.20292.

23. Masheb RM, Grilo CM. Emotional overeating and its associations with eating disorder psychopathology among overweight patients with binge eating disorder. Int J Eat Disord. 2006;39(2):141-6. https://doi.org/10. 1002/eat.20221.

24. Dingemans A, Danner U, Parks M. Emotion regulation in binge eating disorder: a review. Nutrients. 2017;9(11):1274. https://doi.org/10.3390/ nu9111274.

25. Zabala MJ, Macdonald P, Treasure J. Appraisal of caregiving burden, expressed emotion and psychological distress in families of people with eating disorders: a systematic review. Eur Eat Disord Rev. 2009;17(5):33849. https://doi.org/10.1002/erv.925.

26. Johnson F, Wardle J. Dietary restraint, body dissatisfaction, and psychological distress: a prospective analysis. J Abnorm Psychol. 2005;1 14(1):119-25. https://doi.org/10.1037/0021-843X.114.1.119.

27. Leehr EJ, Krohmer K, Schag K, Dresler T, Zipfel S, Giel KE. Emotion regulation model in binge eating disorder and obesity - a systematic review. Neurosci Biobehav Rev. 2015;49:125-34. https://doi.org/10.1016/j.neubi orev.2014.12.008.

28. Morton C, Mooney TA, Lozano LL, Adams EA, Makriyianis HM, Liss M. Psychological inflexibility moderates the relationship between thin-ideal internalization and disordered eating. Eat Behav. 2020;36:101345. https:// doi.org/10.1016/j.eatbeh.2019.101345.

29. Hayes SC, Luoma JB, Bond FW, Masuda A, Lillis J. Acceptance and commitment therapy: model, processes and outcomes. Behav Res Ther. 2006;44(1):1-25. https://doi.org/10.1016/j.brat.2005.06.006.

30. Merwin RM, Timko CA, Moskovich AA, Ingle KK, Bulik CM, Zucker NL. Psychological inflexibility and symptom expression in anorexia nervosa. Eat Disord. 2010;19(1):62-82. https://doi.org/10.1080/10640266.2011.533606.

31. Sandoz EK, Wilson KG, Merwin RM, Kellum KK. Assessment of body image flexibility: the body image-acceptance and action questionnaire. J
Context Behav Sci. 2013;2(1-2):39-48. https://doi.org/10.1016/j.jcbs.2013. 03.002.

32. Duarte C, Ferreira C, Pinto-Gouveia J, Trindade I, Martinho A. What makes dietary restraint problematic? Development and validation of the Inflexible Eating Questionnaire. Appetite. 2017;1 14:146-54. https://doi.org/10. 1016/j.appet.2017.03.034.

33. Tang C, Cooper M, Wang S, Song J, He J. The relationship between body weight and dietary restraint is explained by body dissatisfaction and body image inflexibility among young adults in China. Eat Weight Disord Stud Anorex Bulim Obes. 2020;26:1-8. https://doi.org/10.1007/ s40519-020-01032-0.

34. Pellizzer ML, Waller G, Wade TD. Body image flexibility: a predictor and moderator of outcome in transdiagnostic outpatient eating disorder treatment. Int J Eat Disord. 2018;51(4):368-72. https://doi.org/10.1002/ eat.22842.

35. Wendell JW, Masuda A, Le JK. The role of body image flexibility in the relationship between disordered eating cognitions and disordered eating symptoms among non-clinical college students. Eat Behav. 2012;13(3):240-5. https://doi.org/10.1016/j.eatbeh.2012.03.006.

36. Coimbra M, Ferreira C. Making the leap from healthy to disordered eating: the role of intuitive and inflexible eating attitudes in orthorexic behaviours among women. Eat Weight Disord Stud Anorex Bulim Obes. 2020;26:1-8. https://doi.org/10.1007/s40519-020-00998-1.

37. Barnhart WR, Hamilton L, Jordan AK, Pratt M, Musher-Eizenman DR. The interaction of negative psychological well-being and picky eating in relation to disordered eating in undergraduate students. Eat Behav. 2021;40:101476. https://doi.org/10.1016/j.eatbeh.2021.101476.

38. Shi R, Conrad SA. Correlation and regression analysis. Ann Allergy Asthma Immunol. 2009;103(4 Suppl 1):S35-41. https://doi.org/10.1016/s10811206(10)60820-4.

39. Osborne JW, Waters E. Four assumptions of multiple regression that researchers should always test. Pract Assess Res Eval. 2002;8(1):2.

40. McLean SA, Paxton SJ, Wertheim EH. Factors associated with body dissatisfaction and disordered eating in women in midlife. Int J Eat Disord. 2010;43(6):527-36. https://doi.org/10.1002/eat.20737.

41. Haase AM. Weight perception in female athletes: associations with disordered eating correlates and behavior. Eat Behav. 2011;12(1):64-7. https:// doi.org/10.1016/j.eatbeh.2010.09.004.

42. Collins M, Quinton S. The inter-relationship between diet, selflessness, and disordered eating in Australian women. Brain Behav. 2020;10(9):e01774. https://doi.org/10.1002/brb3.1774.

43. Stice $E$, Desjardins $C D$. Interactions between risk factors in the prediction of onset of eating disorders: exploratory hypothesis generating analyses. Behav Res Ther. 2018;105:52-62. https://doi.org/10.1016/j.brat.2018.03. 005.

44. Kraemer HC, Stice E, Kazdin A, Offord D, Kupfer D. How do risk factors work together? Mediators, moderators, and independent, overlapping, and proxy risk factors. Am J Psychiatry. 2001;158(6):848-56. https://doi. org/10.1176/appi.ajp.158.6.848.

45. Shatte AB, Hutchinson DM, Teague SJ. Machine learning in mental health: a scoping review of methods and applications. Psychol Med. 2019;49(9):1426-48. https://doi.org/10.1017/S0033291719000151.

46. Astorino A, Berti R, Astorino A, Bitonti V, De Marco M, Feraco V, Palumbo A, Porti F, Zannino I. Early detection of eating disorders through machine learning techniques. In: International conference on learning and intelligent optimization: 2020. Springer; 2020. p. 33-9. https://doi.org/10.1007/ 978-3-030-53552-0_5.

47. Haynos AF, Wang SB, Lipson S, Peterson CB, Mitchell JE, Halmi KA, Agras WS, Crow SJ. Machine learning enhances prediction of illness course: a longitudinal study in eating disorders. Psychol Med. 2020;51:1-11. https://doi.org/10.1017/S0033291720000227.

48. Linardon J, Messer M, Helms ER, McLean C, Incerti L, Fuller-Tyszkiewicz M. Interactions between different eating patterns on recurrent binge-eating behavior: a machine learning approach. Int J Eat Disord. 2020;53(4):53340. https://doi.org/10.1002/eat.23232.

49. Pike KM, Hoek HW, Dunne PE. Cultural trends and eating disorders. Curr Opin Psychiatry. 2014;27(6):436-42. https://doi.org/10.1097/YCO.00000 00000000100.

50. Kessler R, Mroczek D. Final versions of our non-specific psychological distress scale. Ann Arbor: Survey Research Center of the Institute for Social Research, University of Michigan; 1994. 
51. Kang Y, Guo W, Xu H, Chen Y, Li X, Tan Z, Li N, Gesang Z, Wang Y, Liu C. The 6-item Kessler psychological distress scale to survey serious mental illness among Chinese undergraduates: psychometric properties and prevalence estimate. Compr Psychiatry. 2015;63:105-12. https://doi.org/ 10.1016/j.comppsych.2015.08.011.

52. Tie $B, C$ Chen $G$, He J. Validation of the inflexible eating questionnaire in a large sample of Chinese adolescents: psychometric properties and gender-related differential item functioning. Eat Weight Disord. 2021. https://doi.org/10.1007/s40519-021-01239-9.

53. He J, Ma H, Barthels F, Fan X. Psychometric properties of the Chinese version of the Düsseldorf Orthorexia Scale: prevalence and demographic correlates of orthorexia nervosa among Chinese university students. Eat Weight Disord Stud Anorex Bulim Obes. 2019;24(3):453-63. https://doi. org/10.1007/s40519-019-00656-1.

54. Basarkod G, Sahdra B, Ciarrochi J. Body image-acceptance and action questionnaire - 5: an abbreviation using genetic algorithms. Behav Ther. 2018;49(3):388-402. https://doi.org/10.1016/j.beth.2017.09.006.

55. Mancuso SG. Body image inflexibility mediates the relationship between body image evaluation and maladaptive body image coping strategies. Body Image. 2016;16:28-31. https://doi.org/10.1016/j.bodyim.2015.10. 003.

56. Ferreira C, Trindade IA, Martinho A. Explaining rigid dieting in normalweight women: the key role of body image inflexibility. Eat Weight Disord Stud Anorex Bulim Obes. 2016;21 (1):49-56. https://doi.org/10.1007/ s40519-015-0188-X.

57. He J, Cai Z, Chen X, Lu T, Fan X. Validation of the Chinese version of the body image acceptance and action questionnaire and the mediating role of body image flexibility in the relationship between body dissatisfaction and psychological distress. Behav Ther. 2021;52(3):539-51. https://doi. org/10.1016/j.beth.2020.07.003.

58. Garner DM, Olmsted MP, Polivy J. Development and validation of a multidimensional eating disorder inventory for anorexia nervosa and bulimia. Int J Eat Disord. 1983;2(2):15-34. https://doi.org/10.1002/1098108X(198321)2:2<15::AID-EAT2260020203>3.0.CO;2-6.

59. Lee S, Lee AM, Leung T, Yu H. Psychometric properties of the Eating Disorders Inventory (EDI-1) in a nonclinical Chinese population in Hong Kong. Int J Eat Disord. 1997;21(2):187-94. https://doi.org/10.1002/(sici) 1098-108x(199703)21:2\%3c187::aid-eat10\%3e3.0.co;2-\#.

60. He J, Sun S, Zickgraf HF, Ellis JM, Fan X. Assessing appetitive traits among Chinese young adults using the adult eating behavior questionnaire: factor structure, gender invariance and latent mean differences, and associations with BMI. Assessment. 2019;28:1073191119864642. https:// doi.org/10.1177/1073191119864642.

61. He J, Latner JD, Wu W, Fan X. Measuring loss of control over eating in a Chinese context: psychometric properties of the full and brief Chinese version of the loss of control over eating scale. Curr Psychol. 2021;40(2):665-74. https://doi.org/10.1007/s12144-018-9976-6.

62. He J, Sun S, Fan X. Validation of the 12-item Short Form of the Eating Disorder Examination Questionnaire in the Chinese context: confirmatory factor analysis and Rasch analysis. Eat Weight Disord. 2021;26(1):201-9. https://doi.org/10.1007/s40519-019-00840-3.

63. Gideon N, Hawkes N, Mond J, Saunders R, Tchanturia K, Serpell L. Development and psychometric validation of the EDE-QS, a 12 Item Short Form of the Eating Disorder Examination Questionnaire (EDE-Q). PLoS ONE. 2016;11(5):e0152744. https://doi.org/10.1371/journal.pone.0152744.

64. Prnjak K, Mitchison D, Griffiths S, Mond J, Gideon N, Serpell L, Hay P. Further development of the 12-item EDE-QS: identifying a cut-point for screening purposes. 2020. https://doi.org/10.21203/rs.3.rs-15840/v1.

65. Van Buuren S, Groothuis-Oudshoorn K. mice: multivariate imputation by chained equations in R. J Stat Softw. 2011;45(1):1-67. https://doi.org/10. 18637/jss.V045.103.

66. Chicco D. Ten quick tips for machine learning in computational biology. BioData Min. 2017;10(1):35. https://doi.org/10.1186/s13040-017-0155-3.

67. Lakshmi TJ, Prasad CSR. A study on classifying imbalanced datasets. In: 2014 First international conference on networks \& soft computing (ICNSC2014): 2014. IEEE; 2014. p. 141-5. https://doi.org/10.1109/CNSC. 2014.6906652.

68. Rout N, Mishra D, Mallick MK. Handling imbalanced data: a survey. In: International proceedings on advances in soft computing, intelligent systems and applications. Springer; 2018. p. 431-43. https://doi.org/10. 1007/978-981-10-5272-9_39.
69. Yu L, Zhou N. Survey of imbalanced data methodologies. arXiv preprint arXiv:210402240 (2021).

70. Chawla NV, Bowyer KW, Hall LO, Kegelmeyer WP. SMOTE: synthetic minority over-sampling technique. J Artif Intell Res. 2002;16:321-57. https://doi. org/10.1613/jair.953.

71. Ramezankhani A, Pournik O, Shahrabi J, Azizi F, Hadaegh F, Khalili D. The impact of oversampling with SMOTE on the performance of 3 classifiers in prediction of type 2 diabetes. Med Decis Mak. 2016;36(1):137-44. https://doi.org/10.1177/0272989X14560647.

72. Potharaju SP, Sreedevi M. An improved prediction of kidney disease using SMOTE. Indian J Sci Technol. 2016;9(31):1-7. https://doi.org/10.17485/ijst/ 2016/v9i31/95634.

73. Feurer M, Klein A, Eggensperger K, Springenberg JT, Blum M, Hutter F. Auto-sklearn: efficient and robust automated machine learning. In: Automated machine learning. Cham: Springer; 2019. p. 113-34. https:// doi.org/10.1007/978-3-030-05318-5_6.

74. Navlani A. Decision tree classification in Python. New York: Data Camp; 2018.

75. Han J, Pei J, Kamber M. Data mining: concepts and techniques. Amsterdam: Elsevier; 2011.

76. Juarascio A, Shaw J, Forman E, Timko CA, Herbert J, Butryn M, Bunnell D, Matteucci A, Lowe M. Acceptance and commitment therapy as a novel treatment for eating disorders: an initial test of efficacy and mediation. Behav Modif. 2013;37(4):459-89. https://doi.org/10.1177/0145445513 478633.

77. Cardoso A, Oliveira S, Ferreira C. Negative and positive affect and disordered eating: the adaptive role of intuitive eating and body image flexibility. Clin Psychol. 2020;24(2):176-85. https://doi.org/10.1111/cp.12224.

78. Ferreira C, Trindade IA, Martinho A. Explaining rigid dieting in normalweight women: the key role of body image inflexibility. Eat Weight Disord. 2016;21 (1):49-56. https://doi.org/10.1007/s40519-015-0188-x.

79. Sandoz E, Wilson K, DuFrene T. Acceptance and commitment therapy for eating disorders: a process-focused guide to treating anorexia and bulimia. Oakland: New Harbinger Publications; 2011.

80. Hyes S, Luoma B, Bond F. Acceptance and commitment therapy: model, process and out comes. Behav Res Ther. 2006;44:1-25. https://doi.org/10. 1016/j.brat.2005.06.006.

81. Masuda A, Le J, Cohen LL. The role of disordered-eating cognitions and psychological flexibility on distress in Asian American and European American college females in the United States. Int J Adv Couns. 2014;36(1):30-42. https://doi.org/10.1007/s10447-013-9188-6.

82. Masuda A, Price M, Anderson PL, Wendell JW. Disordered eating-related cognition and psychological flexibility as predictors of psychological health among college students. Behav Modif. 2010;34(1):3-15. https:// doi.org/10.1177/0145445509351569.

83. Gloster AT, Meyer AH, Lieb R. Psychological flexibility as a malleable public health target: evidence from a representative sample. J Context Behav Sci. 2017;6(2):166-71. https://doi.org/10.1016/j.jcbs.2017.02.003.

84. Tylka TL. The relation between body dissatisfaction and eating disorder symptomatology: an analysis of moderating variables. J Couns Psychol. 2004;51(2):178. https://doi.org/10.1037/0022-0167.51.2.178.

85. Timko CA, Juarascio AS, Martin LM, Faherty A, Kalodner C. Body image avoidance: an under-explored yet important factor in the relationship between body image dissatisfaction and disordered eating. J Context Behav Sci. 2014:3(3):203-11. https://doi.org/10.1016/j.jcbs.2014.01.002.

86. Morgan JF, Reid F, Lacey JH. The SCOFF questionnaire: assessment of a new screening tool for eating disorders. BMJ. 1999;319(7223):1467-8. https://doi.org/10.1136/bmj.319.7223.1467.

87. Krug I, Linardon J, Greenwood C, Youssef G, Treasure J, Fernandez-Aranda F, Karwautz A, Wagner G, Collier D, Anderluh M. A proof-of-concept study applying machine learning methods to putative risk factors for eating disorders: results from the multi-centre European project on healthy eating. Psychol Med. 2021. https://doi.org/10.1017/S003329172100489X.

\section{Publisher's Note}

Springer Nature remains neutral with regard to jurisdictional claims in published maps and institutional affiliations. 